\title{
A Multicenter, Randomized, Double-Blinded, Comparative Study of Two Hyaluronic Acid Filler Products for the Correction of Nasolabial Folds
} \author{
Seongjae Youn ${ }^{8}$, Boncheol Leo Goo ${ }^{9}$ and Kwang Ho Han ${ }^{10 *}$ \\ ${ }^{1}$ Charité Universitätsmedizin, Berlin, Germany \\ ${ }^{2}$ Leaders Aesthetic Laser and Cosmetic Surgery Center, Seoul, Korea \\ ${ }^{3}$ Banobagi Dermatologic Clinic, Seoul, Korea \\ ${ }^{4}$ Apkoo-jung Oracle Dermatology \& Plastic Surgery Center, Seoul, Korea \\ ${ }^{5}$ CNP Skin Clinic, Asan, Korea \\ ${ }^{6}$ Lisienne Skin Clinic, Bundang, Korea \\ 7 JMO Dermatology, Seoul, Korea \\ ${ }^{8}$ Leaders Aesthetic Laser and Cosmetic Surgery Center, Seoul, Korea \\ ${ }^{9}$ Naeum Dermatology and Aesthetics Clinic, Seoul, Korea \\ ${ }^{10}$ Nature Skin Clinic, Seoul, Korea
}

Jihae Yoon ${ }^{1}$, Nark Kyoung Rho' ${ }^{2}$ Heedae Jeon ${ }^{3}$, Je Young Park' ${ }^{4}$ Hyun Jo Kim ${ }^{5}$ Dongjin Im ${ }^{6}$, Wooseok Koh ,

Submission: July 23, 2020; Published: August 03, 2020

*Corresponding author: Kwang Ho Han, Nature Skin Clinic, Seoul, Republic of Korea

\section{Abstract}

Background: Injecting hyaluronic acid (HA) filler has become the most frequently performed minimally invasive procedure for the correction of the nasolabial fold (NLF). Understanding the property of a specific filler product is important when using HA fillers for NLFs since they differ among individuals in shape and dynamics.

Objectives: To compare the efficacy and safety of two commercial HA filler products for the correction of NLFs

Patients/Methods: This is a randomized, multicenter, double-blind, split-face study involving 21 Korean subjects with varying degrees of NLFs. Subjects were randomized to assign the injection with a $24 \mathrm{mg} / \mathrm{mL}$ monophasic HA gel (YS24) and a $20 \mathrm{mg} / \mathrm{mL}$ biphasic HA gel (RP20) to each NLF. Blinded independent evaluators assessed the wrinkle severity rating scale (WSRS) at 3,12, and 24 weeks after injection. Subjects and treating investigators assessed the global aesthetic improvement scale (GAIS) at 3, 12, and 24 weeks. Any adverse events were assessed during the entire study period.

Results: The baseline WSRS scores were $3.29 \pm 0.56$ for both NLFs. At week 24, the mean WSRS score in YS24- and RP20-treated side were $2.29 \pm 0.64$ and $2.33 \pm 0.66$, respectively. There was no statistically significant different change between YS24- and RP20-treated sides. At week 24, the mean GAIS scores of YS24- and RP20-side were $2.10 \pm 0.77$ and $1.57 \pm 0.81$, respectively. Adverse events related to the local injection site were temporary and self-limited. No serious adverse effects were observed during the entire follow-up period.

Conclusion: Both HA filler products were found to be safe and effective for the correction of NLFs. The results of this spilt-face designed study confirm that YS24 is non-inferior to RP20 in correcting moderate to severe NLFs of Korean subjects.

Keywords: Nasolabial Fold; Hyaluronic Acid Filler; Aesthetic Laser; Hyaluronic Acid, Lidocaine

\section{Introduction}

The nasolabial fold (NLF) becomes prominent during the aging process mainly due to the volume loss of deep and superficial soft tissue and redistribution of fat compartments [14]. The deepening of NLF is considered to be one of the challenges 
to correct as the interest of facial rejuvenation grew [5]. Correcting NLFs not only creates a facial V-shape which results in healthy and youthful appearance but also has beneficial effects on neighboring esthetic units [6]. Various filling materials are being used for volume restoration which is essential to correct the NLF [7]. Both effectiveness and safety must be guaranteed when choosing a filling material. It should be low-cost and have acceptable persistency [8]. Among various injection materials, cross-linked hyaluronic acid (HA) gel is considered as a standard filling agent since it has exhibited unique and attractive properties [8]. HA is a ubiquitous component of all mammalian connective tissues therefore it is biocompatible, biodegradable, and non-immunogenic. Injection of HA fillers gives its volume by drawing water and it is also known to stimulate collagen and elastin leading to long-term correcting effect [8-10]. The existence of hyaluronidase attributes to its safety when removal is necessary [8,11]. Therefore, HA fillers have been extensively used as a non-surgical, minimally invasive method to restore nasolabial folds in the past decades. Overall patient satisfaction plays an essential role in a successful treatment in actual practice, thereby it has been incorporated in recent clinical trials $[12,13]$.

Several factors attribute to the level of satisfaction, which includes immediate and extended aesthetic outcomes, patient expectation, length and severity of posttreatment side effects, and technical skills of a physician [13,14]. Thus, injectors should be able to understand the patient's needs and expectations as wells as to set both realistic and satisfying goals. Moreover, it is crucial to choose a proper HA filler product with respective chemical and physical properties since product characteristics are closely related to the level of the patient's satisfaction after injection [13]. One of the most well-known and representative filler products utilizing cross-linked HA is Restylane $\AA$ Perlane (RP20; Galderma SA Lausanne, Switzerland). RP20 is a well-studied HA filler derived from Streptococcus equi. It is a transparent gel with a concentration of $20 \mathrm{mg} / \mathrm{mL}$ suspended in physiological saline ( $\mathrm{pH} 7$ ). It is categorized as a biphasic product consisting of cross-linked HA gels suspended in free HA used as a carrier. Youthfill@ Shape (YS24; RFBio, Seoul, Korea) is a novel HA filler of high density. After extraction and purification, HA is cross-linked with a binding agent 1,4-butanediol diglycidyl ether (BDDE) and reconstituted in a physiologic buffer at $\mathrm{pH} 7$ and concentration of $24 \mathrm{mg} / \mathrm{mL}$. The high concentration of YS24 results in a cohesive gel with high elasticity. It falls into the category of the monophasic filler which consists of a homogeneous mixture of high- and lowmolecular-weight HA, providing good tissue integration. Both fillers contain $0.3 \%$ of lidocaine for the patient's comfort during injection. Considering its high elasticity, we expected YS24 to be an appropriate candidate in treating deep NLFs. This study aimed to investigate the efficacy and safety of YS24 and its non-inferiority compared to a well-studied injectable HA product RP20 to correct NLFs with varying degrees.

\section{Materials and Methods}

\section{Patient selection}

This randomized, double-blinded, and split-face clinical study was conducted at 5 dermatology outpatient clinics (Apgujeong Leaders Dermatology Clinic, Apgujeong Oracle Dermatology Clinic, Banobagi Dermatology Clinic, Cheonan CNP Skin Clinic, Leaders Aesthetic Laser \& Cosmetic Surgery Center, and Lisienne Dermatology Clinic) in South Korea. The study participants were healthy Korean adults aged between 20 to 60 with moderate to severe nasolabial folds [ 3 to 4 points on the Wrinkle Severity Rating Scale (WSRS)], which were evaluated by treating investigators to be approximately symmetrical. All patients participated voluntarily, and all provided written informed consent after a full explanation of the risks and benefits of the procedure. Patients agreed to refrain from any other facial cosmetic procedures during this study period. Patients who showed hypersensitivity to hyaluronic acid, lidocaine, or local anesthetics, were excluded. Also excluded were participants who had undergone laser or chemical skin resurfacing, botulinum toxin injections, facelift procedures, or tissue augmentation in the nasolabial folds within the previous 24 weeks. Participants who were pregnant or not willing to use reliable contraception while participating in the study were excluded.

\section{Study design}

At the initial treatment visit (Week 0), the participants were randomized to determine which NLF, right or left, would receive YS24; RP20 was injected into the contralateral NLF. After the injections, the participants were observed for 30 minutes to check for adverse events. The response to the initial injection of YS24 and RP20 was evaluated at week 3. Touch-up injections were not provided during the study period. The participants were then followed up at 12 and 24 weeks after the initial treatment. Standard clinical photographs were taken at each visit. Additional 3-dimensional images were acquired by the use of a stereo-photogrammetric imaging system (Vectra ${ }^{\circledR}$ M3; Canfield Scientific Inc., Parsippany, NJ). Both participants and evaluating investigators were blinded to the treatments.

\section{Treatment}

With an upright position of the participants, each product was injected with a $25 \mathrm{G}$ blunt-tip cannula. The syringe was inserted in the subdermal plane and the filler was injected as the syringe was withdrawn. When this process was not sufficient to produce enough augmentation, multiple small boluses of filler were injected along the fold. The amount injected was at the discretion of the treating investigators according to the width, length, and depth of the NLF. The mean injected volume per participant was similar for both products and did not exceed $1.5 \mathrm{~mL}$ on any NLF.

\section{Efficacy measures}

The primary objective of this study was to determine the non- 
inferiority of YS24 to RP20 for the correction of NLFs. The primary efficacy measurement was the WSRS scores assessed by the evaluating investigators at week 24 compared to baseline WSRS scores. The secondary efficacy endpoints were (1) evaluating investigator-assessed WSRS scores at weeks 3, 12, and 24; (2) Changes in global aesthetic improvement scale (GAIS) scores measured through participants and treating investigators at weeks 3,12 , and 24; (3) proportion of participants with an improvement of $\geq 1$ grade in the evaluating and treating investigators-assessed WSRS scores over 24 week period. The five-point scale of WSRS represents wrinkle severity: "none" (1 point), "mild" (2), "moderate" (3), "severe" (4), "extreme" (5). GAIS scores measure the improvement on a five-point scale: "worsened" (-1 point), "no change" (0), "improved" (1), "much improved" (2), "very much improved" (3). The pretreatment photograph of each participant served as the reference image at each follow-up visit.

Patients scored the severity of pain during filler injection using a 4-point scale: "none" (0 points), "mild" (1). "moderate" (2), "severe" (3), "extreme" (4). Patients assessed the foreign body sensation immediately after filler injection using a 4-point scale: "none" (0 points), "mild" (1). "moderate" (2), "severe" (3), "extreme" (4). Besides, the patient's satisfaction of volume augmentation was measured immediately after filler injection using a 4-point scale: "very dissatisfied" (0 points), "somewhat dissatisfied" (1), "neutral" (2), "somewhat satisfied" (3), "very satisfied" (4). Volume changes from 3-dimensional images were visualized using the Vectra ${ }^{\circledR}$ Analysis Module software (version 6.1.8, Canfield Scientific Inc., Parsippany, NJ). Image time points included pretreatment, 3 weeks, 12 weeks, and 24 weeks after the injection session.

\section{Safety assessment}

Injection sites were examined by treating investigators for 30 minutes after injection. At each follow-up visit, participants were asked about adverse events (AEs), and the treating investigators examined the injection site for AEs such as erythema, swelling, induration, mass, hematoma, irritation, pruritus, pain, and signs of vascular compromise.

\section{Statistical analysis}

The primary efficacy endpoint was the WSRS score assessed by the evaluating physicians (Koh, Han, and Youn) at week 24. The non-inferiority was declared if the lower limit of the $97.5 \%$ one-tailed interval was greater than -0.29 in the full analysis set. The secondary efficacy endpoints included (1) the WSRS score assessed by the evaluating and treating physicians at each visit (week 12 and 24 for evaluating, week 3, 12, and 24 for treating physicians), (2) the GAIS score assessed by the participants and treating physicians at each visit (week 3, 12 and 24), (3) proportion of subjects with changes of more than 1 point on the WSRS assessed by the evaluating and treating assessors. The statistical test for secondary endpoint was considered significant with a p-value of less than $5 \%$ in a two-tailed $95 \%$ confidence interval.

\section{Results}

\section{Patient demographics and clinical characteristics}

A total of 21 Korean subjects were enrolled and randomized in this clinical study. Nineteen female subjects and two male subjects completed a 24-week follow-up period. The mean ages of the subjects were 39.4 years. The mean injected volumes of YS24 was $1.00 \pm 0.26 \mathrm{~mL}$. The mean injected volumes of RP20 was $1.01 \pm$ $0.25 \mathrm{~mL}$.

\section{Effectiveness}

The baseline WSRS scores (week 0) assessed by evaluating investigators were $3.29 \pm 0.56$ for both NLFs. There were no differences in baseline WSRS scores between the YS24-treated side and the RP20-treated side. At week 24, the mean WSRS score assessed by evaluating investigators in the YS24-treated side and RP20- treated side was $2.29 \pm 0.64$ and $2.33 \pm 0.66$, respectively. There were no statistically significant changes between both sides at week 24 (Figure 1). In the YS24- treated side, 85.7\% (18 of 21) subjects were assessed by evaluating investigator with changes of more than 1 point on the WSRS over 24 weeks. In the RP20-treated side, $80.9 \%$ (17 of 21 ) subjects were assessed by evaluating investigator with changes of more than 1 point on the WSRS over 24 weeks. The mean GAIS score immediately after injection assessed by treating investigators was $2.57 \pm 0.60$ for both NLFs. However, mean GAIS scores assessed by treating investigators at week 24 in the YS24-treated side and the RP20-treated side were $2.10 \pm 0.77$ and $1.57 \pm 0.81$, respectively ( $p$ value 0.211 ). Sixteen of 21 patients scored 'much improved' in the YS24-treated side at week 24, while 10 of 21 patients scored in the RP20-treated side (Figure 2).

The pain score during filler injection was similar in both NLFs (YS24: $0.81 \pm 0.68$, RP20: $0.81 \pm 0.60$ ) Most participants reported the pain severity as none to mild $(0 \sim 1$ point) for both NLFs. The foreign body sensation right after the filler injection was respectively scored on $0.71 \pm 0.64$ and $0.62 \pm 0.59$, in the YS24treated side and RP20-treated side. Most participants assessed the scores of foreign body sensation as none to mild ( $0 \sim 1$ point $)$ for both NLFs. The subjects scored their satisfaction with volume augmentation immediately after filler injection. The scores were $3.71 \pm 0.56$ (YS24) and $3.48 \pm 0.81$ (RP20), respectively. Most participants reported the satisfaction score of either 'somewhat satisfied' ( 3 points) or 'very satisfied' ( 4 points) for both NLFs (Figure 3).

\section{Safety}

Lumpiness ( 4 subjects), erythema ( 1 subject), and swelling ( 1 subject) were noted at the filler injection site. All local AEs were transient and. No late AEs were found at week 24. Both YS24 and RP20 were well tolerated (Figures 4 \& 5). 


\section{WSRS}

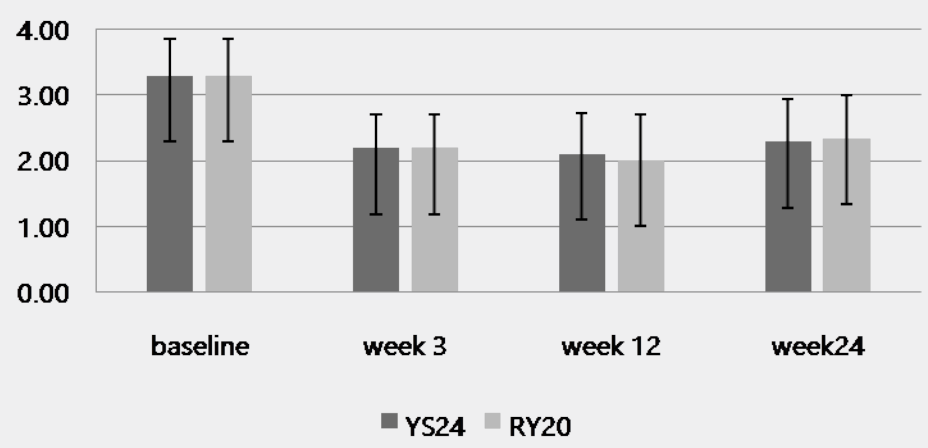

Figure 1: Wrinkle severity rating scale (WSRS) scores assessed by evaluating investigators over 24 weeks after the initial treatment (baseline) with Youthfill ${ }^{\circ}$ Shape (YS24) and Restylane ${ }^{\circledR}$ Perlane (RP20).

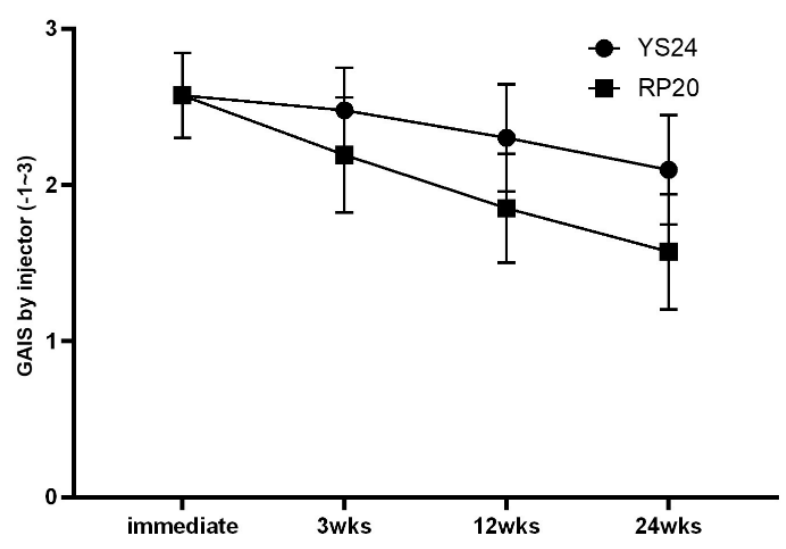

Figure 2: Global aesthetic improvement scale (GAIS) scores evaluated by the treating investigators. (over 24 weeks. GAIS YS24: Youthfill Shape, RP20: Restylane ${ }^{\circledR}$ Perlane).

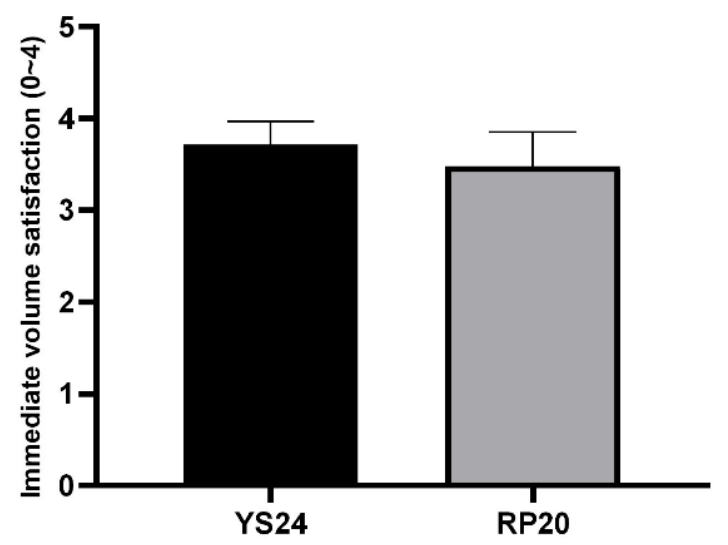

Figure 3: The patient's satisfaction score of volume augmentation immediately after filler injection. (YS24: Youthfill@ Shape, RP20: Restylane ${ }^{\circledR}$ Perlane). 


\section{Juniper Online Journal of Dermatology \& Cosmetics}

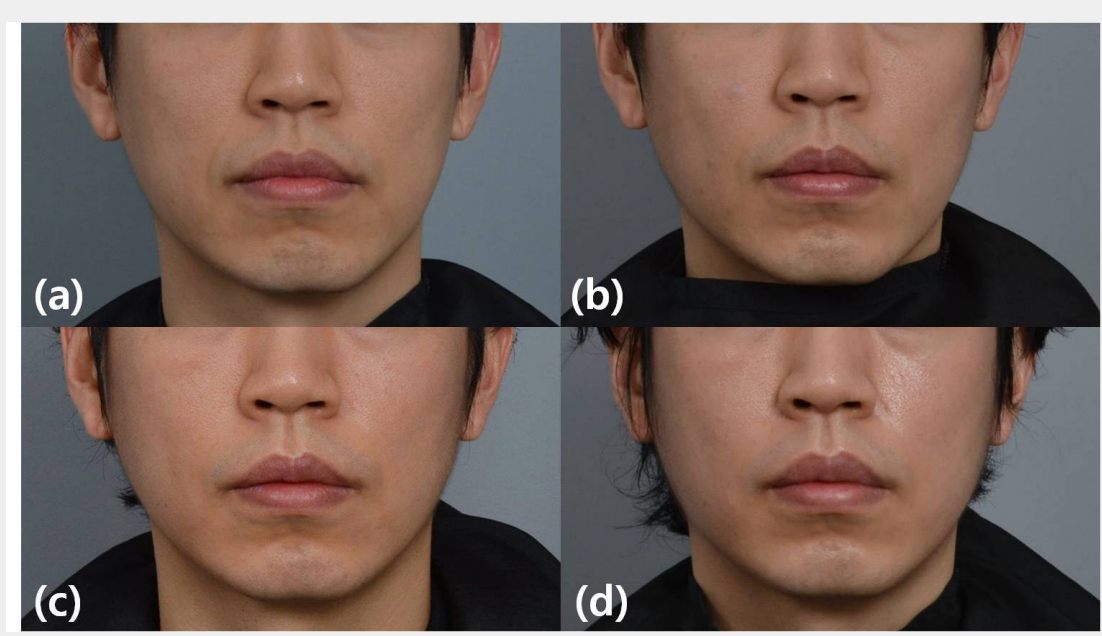

Figure 4(1): Photographic images of a representative participant (30-year-old male). (Right nasolabial fold: Youthfill $囚$ Shape / Left nasolabial fold: Restylane $§$ Perlane). (a) baseline, (b) 3 weeks, (c) 12 weeks, (d) 24 weeks.

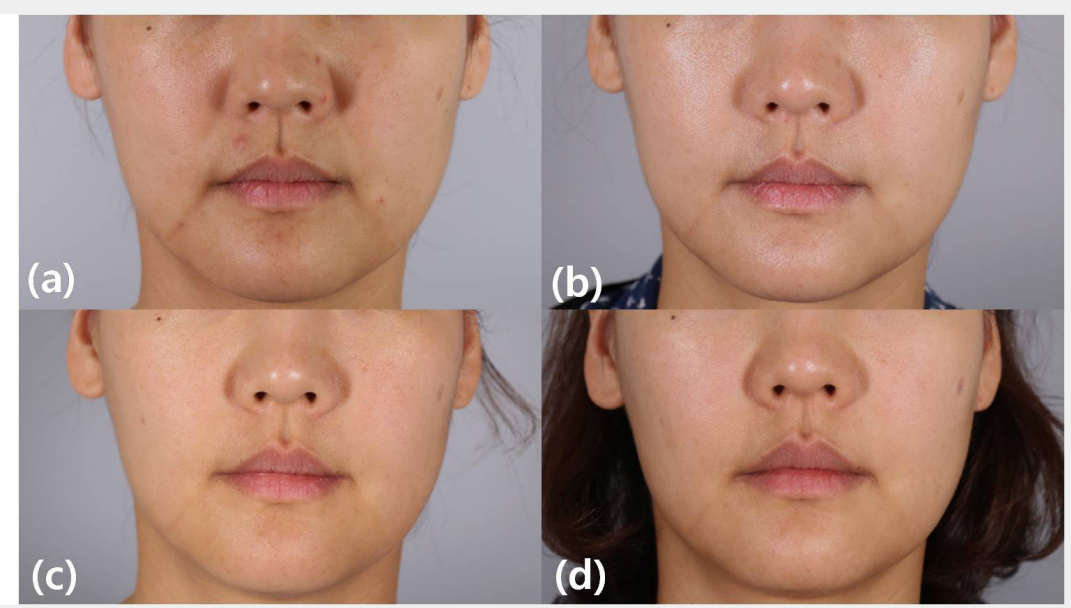

Figure 4(2): Photographic images of a representative participant (41-year-old female). (Right nasolabial fold: Restylane® Perlane / Left nasolabial fold: Youthfill@ Shape). (a) baseline, (b) 3 weeks, (c) 12 weeks, (d) 24 weeks.

\section{Discussion}

In the past decade, the process of aging has been considered as an obstacle to overcome and maintaining the youthful appearance has become a virtue $[5,15]$. In many subjects, the deepening of NLF is related to a loss of upper perioral volume due to the posterior displacement of the maxilla, leaving the facial skin unsupported and tethered by the zygomatic musculature. Therefore, adding volume with fillers not only to the fold but also to the region of the canine fossa helps to restore the youthful midface $[3,8]$. Numerous dermal fillers have been introduced in the market, as the demand for filler injection increased accordingly. Among others, HA fillers are considered to be ideal for this aesthetic need as they are safe, effective, biocompatible, non-immunogenic, and easy to distribute and to correct [8-10]. In this multicenter, randomized, split-face clinical trial, we evaluated the efficacy and safety of newly developed HA filler of YS24 for the correction of NLFs in Korean subjects and compared with those of RP20. YS24 showed non-inferiority in its effectiveness. Both agents were shown to be generally well-tolerated and safe with only a few mild and transient injection-site adverse events.

In our study, subjects and evaluating investigators were blinded, whereas the treating investigators were not. Treating investigators took part in the evaluation of primary and secondary endpoints. The primary endpoint (WSRS score at week 24) evaluated by both evaluating and treating investigators at week 24 did not differ with one another and secondary endpoints (GAIS and WSRS scores over 24 weeks) did not show a significant difference among non-blinded treating investigators, blinded participants, and evaluating investigators. One remarkable difference was observed in the GAIS of treating investigators. We speculated 
that the difference in HA concentration could contribute to the superiority of YS24 to RP20. Additionally, the high concentration and monophasic features of YS24 result in a cohesive gel with high elasticity. This cohesiveness and higher elastic modulus $\left(G^{\prime}\right)$ than other HA fillers also lead to this difference of GAIS evaluation. One of the limitations of the present study is the short observation period. The effectiveness and safety were monitored for only 6 months. A longer follow-up period may offer further information about the overall durability of the effect and the long-term safety including delayed-onset AEs.

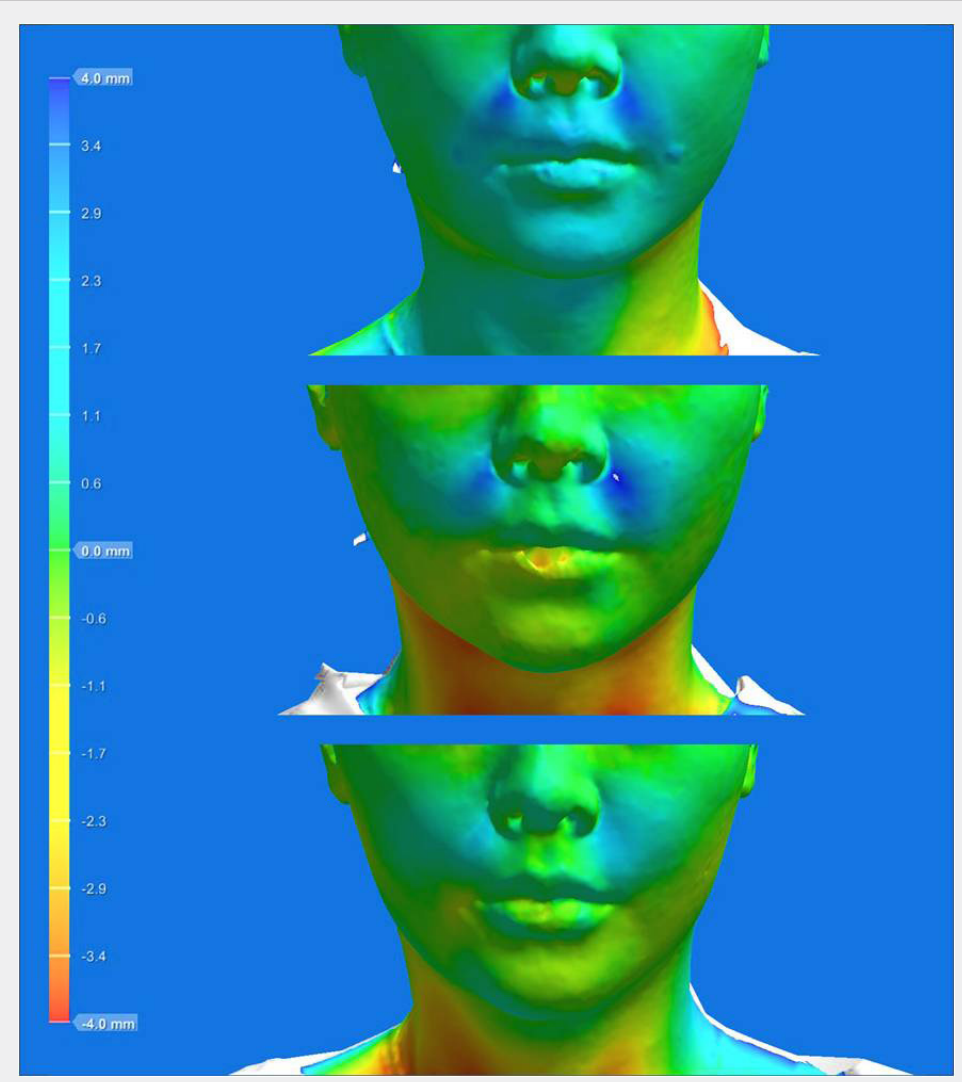

Figure 5: Representative photographs of volume augmentation after filler injection in the nasolabial fold. Subject's nasolabial folds were filled with Restylane ${ }^{\circledR}$ Perlane (right side) and Youthfill囚 Shape (left side). A volume increase was visualized using the 3-dimensional image analysis software, with areas in blue representing increased volume and green no change in volume (upper row: baseline vs 3 weeks, middle row: baseline vs 12 weeks, lower row: baseline vs 24 weeks). All photos were taken with a Vectra M3 imaging system.

\section{Conclusion}

In conclusion, this 24-week clinical trial demonstrated that a novel HA filler YS24 is non-inferior to RP20 in correcting moderate to severe NLFs over 24 weeks in Korean subjects. Further studies with a longer follow-up period are needed to confirm the durability and long-term safety of the product.

\section{References}

1. Cotofana S, Fratila AA, Schenck TL, Redka Swoboda W, Zilinsky I, et al. (2016) The Anatomy of the Aging Face: A Review Facial Plast Surg 32(3): 253-260.

2. Ramesh S, Johnson P, Sarcu D, Wulc AE (2020) Gravity in Midfacial Aging: A 3-Dimensional Study. Aesthet Surg J 24: sjaa021.

3. Wen LH, Zhong PH, Wang XL, An Y, Hu ZQ et al. (2019) Analysis of agerelated changes in midfacial fat compartments in Asian women using computed tomography. J Plast Reconstr Aesthet Surg 72(11): 18391846.

4. Shaw RB, Katzel EB, Koltz PF, Yaremchuk MJ, Girotto JA, et al. (2011) Aging of the facial skeleton: aesthetic implications and rejuvenation strategies. Plast Reconstr Surg 127(1): 374-383.

5. Wu WT, Liew S, Chan HH, Ho WW, Supapannachart N, et al. (2016) Consensus on Current Injectable Treatment Strategies in the Asian Face. Aesthetic Plast Surg 40(2): 202-214.

6. Wollina U (2016) Facial rejuvenation starts in the midface: threedimensional volumetric facial rejuvenation has beneficial effects on nontreated neighboring esthetic units. J Cosmet Dermatol 15(1): 8288.

7. Rubin MG(2004) Treatment of nasolabial folds with fillers. Aesthet Surg J 24(5):489-493.

8. Brandt FS, Cazzaniga A (2008) Hyaluronic acid gel fillers in the management of facial aging. Clin Interv Aging 3(1): 153-159.

9. Fallacara A, Manfredini S, Durini E, Vertuani S (2017) Hyaluronic Acid Fillers in Soft Tissue Regeneration. Facial Plast Surg 33(1): 87-96.

10. Bukhari SNA, Roswandi NL, Waqas M, Habib H, Hussain F, et al. (2018) Hyaluronic acid, a promising skin rejuvenating biomedicine: A review of recent updates and pre-clinical and clinical investigations on cosmetic and nutricosmetic effects. Int J Biol Macromol 120(Pt B): 1682-1695. 
11. Sun ZS, Zhu GZ, Wang HB, Xu X, Cai B, et al. (2015) Clinical Outcomes of Impending Nasal Skin Necrosis Related to Nose and Nasolabial Fold Augmentation with Hyaluronic Acid Fillers. Plast Reconstr Surg 136(4): 434e-441e.

12. Noh TK, Moon HR, Yu JS, Chang SE, Moon IJ, et al. (2016) Effects of highly concentrated hyaluronic acid filler on nasolabial fold correction: A 24-month extension study. J Dermatolog Treat 27(6): 510-514.

13. Fagien S (2010) Maximizing patient satisfaction with facial soft tissue fillers: A question of balance. Cosmetic Dermatology 23(5): 204-212.
14. Arruda LH, Rocha FT, Rocha A (2008) Studying the satisfaction of patients on the outcome of an aesthetic dermatological filler treatment. J Cosmet Dermatol 7(4): 246-250.

15. Liew S, Wu WT, Chan HH, Ho WW, Kim HJ, et al. (2016) Consensus on Changing Trends, Attitudes, and Concepts of Asian Beauty. Aesthetic Plast Surg 40(2): 193-201.

Your next submission with Juniper Publishers
will reach you the below assets
- Quality Editorial service
- Swift Peer Review
- Reprints availability
- E-prints Service
- Manuscript Podcast for convenient understanding
- Global attainment for your research
- Manuscript accessibility in different formats
( Pdf, E-pub, Full Text, Audio)
- Unceasing customer service
Track the below URL for one-step submission
https://juniperpublishers.com/online-submission.php

